\title{
Weaning From Mechanical Ventilation in Patients With Severe Head Trauma: A Proposol of Tracheostomy Antecipation
}

\author{
Daniela Cristina dos Santos Faez ${ }^{\mathrm{a}, \mathrm{c}}$, Carolina Kosour ${ }^{\mathrm{b}}$, Luciana Castilho de Figueiredo ${ }^{\mathrm{a}}$, \\ Rodrigo Marques Tonella ${ }^{a}$, Vania Graner Silva Pinto ${ }^{a}$, Desanka Dragosavac ${ }^{a}$, \\ Antonio Luis Eiras Falcao ${ }^{\mathrm{a}}$
}

\begin{abstract}
Background: The aims of the study were to analyze the withdrawal of mechanical ventilation (MV) of severe head trauma from admitted victims to the adult intensive care unit and to propose a time to perform the tracheostomy, not taking into consideration only the Glasgow coma scale (GCS) but the GCS and the Marshall's computed tomography (CT) scan classification.
\end{abstract}

Methods: This was a cross-sectional, descriptive, observational study, based on continuous registration database collection, medical and physical therapy records. Data were collected from March 2012 to March 2015.

Results: We collected 118 patients. There was no association between GCS of 3 - 5 and 6 - 8, and Marshall's CT classification 3 - 4 and 5 - 6, with extubation, reintubation and tracheostomy. Tracheostomy was performed on $50 \%$ of the population. Regarding the withdrawal of MV after tracheostomy, $62.71 \%$ of patients underwent nebulization protocol $\leq 48$ hours. There was no association between GCS of $3-5$ and 6 - 8, and Marshall's CT classification $3-4$ and 5 - 6, with weaning after tracheostomy.

Conclusions: GCS 3 - 5 tends to be less extubated and has reintubation rate higher than GCS of 6 - 8; the worse the rating of Marshall's CT classification, the lower the extubation rate; and the majority of patients were withdrawn from the ventilator at or less than 48 hours after tracheostomy. We suggest that early tracheostomy, less than 48 hours after intubation, should be performed if clinical and neurological stabilization was achieved in patients with severe traumatic brain injury (TBI) (and Marshall's CT classification III and IV).

Keywords: Brain injuries; Ventilator weaning; Intensive care units; Physical therapy specialty

Manuscript accepted for publication June 08, 2016

aCampinas State University, UNICAMP, Brazil

${ }^{\mathrm{b}}$ Alfenas Federal University, Brazil

${ }^{\mathrm{c} C o r r e s p o n d i n g ~ A u t h o r: ~ D a n i e l a ~ C r i s t i n a ~ d o s ~ S a n t o s ~ F a e z, ~ V i t a l ~ B r a s i l ~ S t r e e t, ~}$ 251, Cidade Universitaria Zeferino Vaz, Campinas, SP, CEP: 13083-888, Brazil.Email: danifaez@gmail.com

doi: http://dx.doi.org/10.14740/jnr380w

\section{Introduction}

Traumatic brain injury (TBI) is the biggest socioeconomic and public health problem in the world [1-6].

To define the severity of the brain injury and the appropriate treatment, computed tomography (CT), based on Marshall's classification [7], and the Glasgow coma scale (GCS) [8] are the tools used upon hospital admission [2].

Generally, the complete neurological damage after severe TBI does not happen at the time of the trauma itself, but after a few minutes, hours or days [9]. The primary lesion may be aggravated by secondary injury, especially in the presence of hypoxia [10-12].

The focus of multidisciplinary care is based on prevention and limitation of secondary brain damage, and the priority is to provide the best possible conditions to favor the recovery of brain lesions. For better control of ventilation, oxygenation and respiratory protection, intubation, sedation and mechanical ventilation (MV) is often required. The adoption of any of these strategies can occur at any time during treatment $[2,13$, 14].

After clinical stabilization and control of intracranial pressure after primary neurological damage, the transition from control ventilation to spontaneus ventilation begins $[15,16]$.

The weaning process from MV involves the reduction of ventilatory parameters and extubation $[17,18]$. Daily, careful evaluation of clinical and neurological conditions and completion of spontaneous breathing trial (SBT) should be considered in order to recognize and facilitate the process of withdrawal of the MV [19].

It is defined as extubation success when the ventilatory prosthesis is removed (extubation) after the patient passed the SBT and there is no need for reinstitution of the MV in the next $48 \mathrm{~h}$. In cases of tracheostomy patients, extubation success occurs when disconnecting the MV after the SBT and there is no need to reconnect to the MV in the next $48 \mathrm{~h}$ [18-20].

About $5-20 \%$ of individuals, even with recovery from acute brain injury, can not proceed to ventilatory weaning and extubation process. These individuals often develop dependence on the MV, and this dependence increases the length of stay and the likelihood of infections related to health care. When this occurs, a tracheostomy is performed [15, 20-22]. However, the ideal moment to perform the tracheostomy is 
Table 1. General Characteristics of Patients With Severe TBI and Mortality Rates

\begin{tabular}{|c|c|c|c|c|c|c|c|c|}
\hline Data & Frequency & $\%$ & Median & Standard deviation & Median & Q1 & Q3 & P value \\
\hline Age & 118 & & 38.09 & 15.22 & 37.0 & 26.0 & 45.5 & - \\
\hline Male & 102 & $86.44 \%$ & - & - & - & - & - & - \\
\hline Mortality rate GCS 6 - 8 & - & $26.67 \%$ & - & - & - & - & - & \\
\hline Mortality rate Marshall's CT III & - & $33.33 \%$ & - & - & - & - & - & 0.41 \\
\hline Mortality rate Marshall's CT VI & - & $53.33 \%$ & - & - & - & - & - & \\
\hline
\end{tabular}

Q1: lower quartile; Q3: upper quartile; GCS: Glasgow coma scale; CT: computed tomography.

still controversial in the literature [8, 23-28].

In 1989, the American College of Chest Physicians advised that tracheostomy should be performed in subjects with proposal for maintenance of endotracheal intubation for at least 21 days [8, 15, 27-31]. The Brazilian Guidelines of MV suggest that tracheostomy should be performed early, up to 7 days in patients with severe TBI [20].

Thus, the objectives of this study were to report the influence of GCS and Marshall's CT scan classification in the weaning process of severe TBI individuals admitted to the intensive care unit (ICU) and, through these observations, we propose a time to perform the tracheostomy not taking into consideration only the GCS but the GCS and the Marshall's CT scan classification.

\section{Methods}

The project was approved by the Research Ethics Committee of the School of Medical Sciences, State University of Campinas, No. 1311/2011. It was a cross-sectional, descriptive and observational study.

Data were collected from ICU continuous registration database, medical and physical therapy records from March 2012 to March 2015.

The study included individual victims of severe TBI, in the acute phase, 18 years, of both sexes, and who were referred to the ICU.

Statistical analysis was performed using SPSS version 13.0 for Windows. The Kolmogorov-Smirnov test was performed to test the normality of the data. Descriptive analysis was performed using graphs and tables for categorical variables and graphs and statistics for numeric variables. Chisquare test was used to analyze categorical variables and when it was not possible to apply, we used the Fisher's exact test. To compare the average of the numerical variables, we used a $t$-test when normality was checked and the Mann-Whitney test for independent samples. $\mathrm{P}$ values $<0.05$ were considered statistically significant.

\section{Results}

From March 2012 to March 2015, 118 patients were involved. The patients' GCS scores were divided into two groups: patients with GCS 6 - 8 (total of 49 subjects), and GCS 3 - 5 (total of 69 subjects). Regarding Marshall's CT scan classification, we evaluated the patients with diffuse lesions class III and IV (total of 55 subjects), and patients with focal lesion class $\mathrm{V}$ and VI (total of 21 individuals). Patients with diffuse lession class I (two patients) and class II (40 patients) were evaluated in another study.

Table 2. Events Related to Weaning From Mechanical Ventilation Associated With the GCS

\begin{tabular}{llll}
\hline Events & Yes & $\mathbf{3 4 8 ~ h}$ & P value \\
\hline Extubation GCS 3 - 5 & $23.26 \%$ & - & 0.21 \\
Extubation GCS 6 - 8 & $34.67 \%$ & - & \\
Reintubation GCS 3 - 5 & $4.76 \%$ & - & 0.61 \\
Reintubation GCS 6 - 8 & $2.67 \%$ & - & \\
Tracheostomy GCS 3 - 5 & $55.81 \%$ & - & 0.44 \\
Tracheostomy GCS 6 - 8 & $46.67 \%$ & - & \\
Weaning MV after tracheostomy GCS 3 - 5 & - & $70.83 \%$ & 0.41 \\
Weaning MV after tracheostomy GCS 6 - 8 & - & $57.14 \%$ & \\
\hline
\end{tabular}

GCS: Glasgow coma scale; MV: mechanical ventilation. 
Table 3. Events Related to Weaning From Mechanical Ventilation Associated With Marshall's CT Scan Classification

\begin{tabular}{lll}
\hline Events & Yes & P value \\
\hline Extubation Marshall's CT III & $29.17 \%$ & 0.08 \\
Extubation Marshall's CT IV & $9.68 \%$ & \\
Extubation Marshall's CT V & $50 \%$ & 0.11 \\
Extubation Marshall's CT VI & $13.33 \%$ & \\
Reintubation Marshall's CT III & $8.33 \%$ & 0.18 \\
Reintubation Marshall's CT IV & $0 \%$ & \\
Reintubation Marshall's CT V & $0 \%$ & 1 \\
Reintubation Marshall's CT VI & $6.67 \%$ & \\
\hline
\end{tabular}

CT: computed tomography.

Table 1 shows the general features of patients and mortality rates associated with GCS 3 - 5 and 6 - 8 and the Marshall's CT scan classification III, IV, V and VI.

\section{Associations with GCS}

Table 2 shows the events related to weaning from MV and its association with GCS 3 - 5 and $6-8$.

\section{Associations with Marshall's CT scan classification}

Tables 3 and 4 show the events related to MV and its association with the Marshall's CT scan classification III, IV, V and VI.

\section{Discussion}

Of the 118 patients, it was observed that most were male and young adults, which corroborates the findings in the literature $[17,28,32]$.

Falcao et al (1995) [33], Dantas Filho et al (2004) [34] and Oliveira et al (2012) [35] conducted studies and found that in relation to prognostic factors in patients with severe TBI, the most influential are the GCS, presence of intracranial hypertension, type of intracranial lesion, presence of hypoxia and hypotension or association between them. They also showed that the initial clinical manifestation is a strong indicator of the severity of primary and secondary lesions associated with TBI. Martins et al (2009) [36] in a prospective study found that patients with GCS 3 or 4 tend to have higher mortality compared to GCS 7 or 8 at hospital admission. In this study, corroborating this statement, although without statistical significance, when we combined GCS and mortality rates, we observed higher mortality rates in severe TBI patients with GCS of 3 - 5 than in those with severe TBI with GCS of 6 - 8 .

Regarding the association of Marshall's CT scan classification and mortality rates, there was a significant difference when compared to mortality in individuals with Marshall's CT scan classification V and VI $(\mathrm{P}=0.04)$. Although without statistical significance, patients with Marshall's CT scan classification IV had a higher mortality rate (45.16\%), than Marshall's CT scan classification III (33.33\%).

These findings corroborate those of Kim (2011) [37], who conducted a systematic review of the literature in order to determine which factors really are prognostic predictors for patients with TBI. She analyzed 46 studies and found as a result that among other factors, a worse Marshall's CT scan classification influences worse prognosis. Contradicting these assertions, Maas et al (2005) [38] conducted a study with the objective of verifying the predictive value of Marshall's CT scan classification. They concluded that for predicting prognosis, the combination predictors of individual items should be preferably performed, such as basal cisterns state, traumatic hemorrhage or subarachnoid hemorrhage and intraventricuar presence of different types of mass lesion instead of Marshall's CT scan classification. In another study, Deepika et al (2015) [39] compared the Marshall's CT scan classification and Rotterdam's scan classification and concluded that both ratings are good for predicting early mortality after moderate to severe TBI, but the classification of Rotterdam may be preferable in cases of diffuse injuries, because it contains additional items such as the presence of subarachnoid hemorrhage.

As part of the treatment and to prevent secondary injury, it is necessary to use MV. All subjects in this study underwent

Table 4. Events Related to Weaning From Mechanical Ventilation Associated With Marshall's CT Scan Classification

\begin{tabular}{llll}
\hline Events & Yes & $\mathbf{3 4 8 ~ h}$ & P value \\
\hline Tracheostomy Marshall's CT III & $45.83 \%$ & - & 0.59 \\
Tracheostomy Marshall's CT IV & $54.84 \%$ & - & \\
Tracheostomy Marshall's CT V & $50 \%$ & - & 1 \\
Tracheostomy Marshall's CT VI & $46.67 \%$ & - & \\
Weaning MV after tracheostomy Marshall's CT III & - & $54.55 \%$ & 0.44 \\
Weaning MV after tracheostomy Marshall's CT IV & - & $70.59 \%$ & \\
Weaning MV after tracheostomy Marshall's CT V & - & $100 \%$ & 0.47 \\
Weaning MV after tracheostomy Marshall's CT VI & - & $57.14 \%$ & \\
\hline
\end{tabular}

MV: mechanical ventilation; CT: computed tomography. 
endotracheal intubation.

After the pre-hospital care, the patients are referred to the hospital and following medical or surgical treatment, the individual are sent to the ICU. When the patients are neurologicaly and hemodynamicaly stabilized, the process of weaning begins.

In relation to extubation, Roquilly et al (2013) [40] concluded that the implementation of an extubation protocol favors the reduction of the MV time of patients with moderate to severe TBI but does not reduce the mortality rate. King et al (2010) [41] reported that it is now understood that many patients who are unable to obey commands, but have the ability to maintain pervious airway can be safely extubated.

Lazaridis et al (2012) [15] in a review of the general principles of the current literature on covering neurosurgical patients with primary brain injury found that the criteria for extubation included a combination of neurological, hemodynamic and respiratory findings, but more studies are needed to better evaluate extubation indicators, extubation failure predictors and determine the most appropriate time for tracheostomy indication for this population. Savi et al (2012) [18] conducted a study which evaluated the potential of predictive indices of successful extubation during the weaning process. The protocol included hemodynamics and MV parameters, arterial blood gas analisys and weaning process. All patients included in the study were extubated after $30 \mathrm{~min}$ of successful SBT. However, the population was not exclusively made up of individuals with TBI.

In the present study, when combined GCS and extubation rates and reintubation, it was observed, although without statistical significance, that patients with GCS $6-8$ tend to be extubated with more frequency than GCS 3 - 5. Moreover, although the reintubation rate was low, it is known that patients with GCS 3 - 5 tend to be more reintubated than GCS $6-8$.

When we studied the association between Marshall's CT scan classification and extubation rates, it was observed that the worse the rating of Marshall's CT scan classification, the lower the extubation rate. Among the patients with Marshall's CT scan classification IV, only $9.68 \%$ were extubated and those with Marshall's CT scan classification VI, only $13.33 \%$ were extubated. When we associated Marshall's CT scan classification and reintubation rates, it was observed that in patients with Marshall's CT scan classification IV, despite the low extubation rate, no patient was reintubated. In the group of patients with Marshall's CT scan classification VI, half of the subjects underwent reintubation. Karanjia et al (2011) [19] conducted a study and found that the main cause of reintubation in patients with primary brain injury is the neurological respiratory failure, which was what happened in the present study patients.

When there is failure in ventilatory weaning and extubation, the tracheostomy is indicated. A study was conducted in trauma patients and it was found that the realization of tracheostomy does not reduce mortality, incidence of pneumonia and laryngotracheal injury but has influence on the reduction of MV time in patients with severe TBI [20]. In the present study, when combined to the GCS and the rate of performing tracheostomy, it was observed that patients with GCS 3 - 5 are more subjected to tracheostomy than the patients GCS $6-8$, although tracheostomy realization rate is high in both groups
(55.81\% and $46.67 \%$, respectively).

Regarding the association of GCS and MV withdraw after completion of tracheostomy, it was observed that independent of the value of the GCS, most were removed from MV in less or equal to $48 \mathrm{~h}$ after completion of tracheostomy. Of the patients GCS $3-5,70.83 \%$ left the MV in less or equal to $48 \mathrm{~h}$ after completion of tracheostomy, which suggests that tracheostomy is beneficial for this population.

When we associated Marshall's CT scan classification and the completion of tracheostomy, it was observed that independent of Marshall's CT scan classification, half of the population underwent tracheostomy. However, most who underwent tracheostomy were those included in Marshall's CT scan classification IV.

Regarding the association of Marshall's CT scan classification and MV withdraw after completion of tracheostomy, in individuals with Marshall's CT scan classification IV, 70\% left the MV in less than or equal to $48 \mathrm{~h}$. Of patients with Marshall's CT scan classification V, everyone left the MV in less than or equal to $48 \mathrm{~h}$. These data lead us to infer that this population would benefit from the realization of tracheostomy early on.

As recommendations, a study has proposed that if the patient requires MV up to 10 days, one can maintain the endotracheal tube. It is known that if there will be a need for MV for more than 21 days, one can indicate the tracheostomy; if you can not determine the period that the individual needs MV, it is suggested that we make a daily assessment to indicate the tracheostomy as soon as possible to minimize the time of endotracheal tube [42].

Some authors [16, 21, 24, 26, 42-46] defend the implementation of early tracheostomy, and show that it reduces the duration of MV, ICU, ventilator-associated pneumonia, induced lung injury by MV, hospital costs and mortality and favors bronchial hygiene, but there is no global consensus on the time that is considered early tracheostomy.

In the present study, it was not analyzed how long after the installation of endotracheal tube the tracheostomy was indicated due to the fact the date indication is not listed in medical records. This fact does not allow us to say how many tracheostomies were indicated early.

After analyzing the results of our study, we suggest that early tracheostomy, less than $48 \mathrm{~h}$ after intubation should be performed if clinical and neurological stabilization was achieved in patients with severe TBI (GCS 3 - 5) and Marshall's CT scan classification III and IV, in order to reduce the duration of MV, ICU length of stay and complications from the use of it, especially pneumonia associated with MV.

\section{Conclusions}

We conclude that patients with severe TBI that present GCS 3 - 5 tend to be less extubated and have reintubation rate higher than those with severe TBI that present GCS 6 - 8. It also follows that the worse Marshall's CT scan classification, the lower the rate extubation.

In our study, the tracheostomy was performed, regardless of GCS and Marshall's CT scan classification, but the worse 
the GCS, the higher the rate of realization.

In relation to weaning of MV after completion of tracheostomy, it is concluded that regardless of the value of the GCS and Marshall's CT scan classification, most patients leave the VM less or equal to $48 \mathrm{~h}$, which suggests that tracheostomy is beneficial to this population and if carried out early on could further reduce the length of stay in ICU, MV complications from the use of it.

We suggest that early tracheostomy, less than $48 \mathrm{~h}$ after intubation should be performed if clinical and neurological stabilization was achieved in patients with severe TBI and Marshall's CT classification III and IV.

\section{Conflicts of Interest}

The authors declare that they have no conflicts of interest.

\section{References}

1. Lingsma HF, Roozenbeek B, Steyerberg EW, Murray GD, Maas AI. Early prognosis in traumatic brain injury: from prophecies to predictions. Lancet Neurol. 2010;9(5):543554.

2. Maas AI, Stocchetti N, Bullock R. Moderate and severe traumatic brain injury in adults. Lancet Neurol. 2008;7(8):728-741.

3. Sharma S, Gomez D, de Mestral C, Hsiao M, Rutka J, Nathens AB. Emergency access to neurosurgical care for patients with traumatic brain injury. J Am Coll Surg. 2014;218(1):51-57.

4. Ladanyi S, Elliott D. Traumatic brain injury: an integrated clinical case presentation and literature review. Part I: assessment and initial management. Aust Crit Care. 2008;21(2):86-95.

5. Herou E, Romner B, Tomasevic G. Acute Traumatic Brain Injury: Mortality in the Elderly. World Neurosurg. 2015;83(6):996-1001.

6. Brorsson C, Rodling-Wahlstrom M, Olivecrona M, Koskinen LO, Naredi S. Severe traumatic brain injury: consequences of early adverse events. Acta Anaesthesiol Scand. 2011;55(8):944-951.

7. Marshall LF, Marshall SB, Klauber MR, Van Berkum Clark M, Eisenberg H, Jane JA, Luerssen TG, et al. The diagnosis of head injury requires a classification based on computed axial tomography. J Neurotrauma. 1992;9(Suppl 1):S287-292.

8. Teasdale G, Jennett B. Assessment of coma and impaired consciousness. A practical scale. Lancet. 1974;2(7872):81-84.

9. PreHospital Emergency Care - Official Journal of the National Association of EMS Physicians; supplement to volume 12; numero 1; Janeiro - Marco; 2007.

10. Winter CD, Adamides AA, Lewis PM, Rosenfeld JV. A review of the current management of severe traumatic brain injury. Surgeon. 2005;3(5):329-337.

11. Lee JC, Rittenhouse K, Bupp K, Gross B, Rogers A, Rogers FB, Horst M, et al. An analysis of Brain Trauma Foun- dation traumatic brain injury guideline compliance and patient outcome. Injury. 2015;46(5):854-858.

12. Ghajar J. Traumatic brain injury. Lancet. 2000;356(9233):923-929.

13. Haddad SH, Arabi YM. Critical care management of severe traumatic brain injury in adults. Scand J Trauma Resusc Emerg Med. 2012;20:12.

14. Walcott BP, Kahle KT, Simard JM. The DECRA trial and decompressive craniectomy in diffuse traumatic brain injury: is decompression really ineffective? World Neurosurg. 2013;79(1):80-81.

15. Lazaridis C, DeSantis SM, McLawhorn M, Krishna V. Liberation of neurosurgical patients from mechanical ventilation and tracheostomy in neurocritical care. J Crit Care. 2012;27(4):417 e411-418.

16. Juern JS. Removing the critically ill patient from mechanical ventilation. Surg Clin North Am. 2012;92(6):14751483.

17. dos Reis HF, Almeida ML, da Silva MF, Moreira JO, Rocha Mde S. Association between the rapid shallow breathing index and extubation success in patients with traumatic brain injury. Rev Bras Ter Intensiva. 2013;25(3):212-217.

18. Savi A, Teixeira C, Silva JM, Borges LG, Pereira PA, Pinto $\mathrm{KB}, \mathrm{Gehm} \mathrm{F}$, et al. Weaning predictors do not predict extubation failure in simple-to-wean patients. J Crit Care. 2012;27(2):221 e221-228.

19. Karanjia N, Nordquist D, Stevens R, Nyquist P. A clinical description of extubation failure in patients with primary brain injury. Neurocrit Care. 2011;15(1):4-12.

20. Brazilian Guidelines of mechanical ventilation, 2013; Available in [http://itarget.com.br/newclients/sbpt.org. br/2011/downloads/arquivos/Dir_VM_2013/Diretrizes VM2013_SBPT_AMIB.pdf]; Accessed in December, 2015.

21. Siddiqui UT, Tahir MZ, Shamim MS, Enam SA. Clinical outcome and cost effectiveness of early tracheostomy in isolated severe head injury patients. Surg Neurol Int. 2015;6:65.

22. Chang WT, Nyquist PA. Strategies for the use of mechanical ventilation in the neurologic intensive care unit. Neurosurg Clin N Am. 2013;24(3):407-416.

23. Huang YH, Lee TC, Liao CC, Deng YH, Kwan AL. Tracheostomy in craniectomised survivors after traumatic brain injury: a cross-sectional analytical study. Injury. 2013;44(9):1226-1231.

24. Alali AS, Scales DC, Fowler RA, Mainprize TG, Ray JG, Kiss A, de Mestral C, et al. Tracheostomy timing in traumatic brain injury: a propensity-matched cohort study. J Trauma Acute Care Surg. 2014;76(1):70-76; discussion 76-78.

25. Rizk EB, Patel AS, Stetter CM, Chinchilli VM, Cockroft KM. Impact of tracheostomy timing on outcome after severe head injury. Neurocrit Care. 2011;15(3):481-489.

26. Shamim MS, Qadeer M, Murtaza G, Enam SA, Farooqi NB. Emergency department predictors of tracheostomy in patients with isolated traumatic brain injury requiring emergency cranial decompression. J Neurosurg. 2011;115(5):1007-1012. 
27. Goettler CE, Fugo JR, Bard MR, Newell MA, Sagraves SG, Toschlog EA, Schenarts PJ, et al. Predicting the need for early tracheostomy: a multifactorial analysis of 992 intubated trauma patients. J Trauma. 2006;60(5):991-996.

28. Hsu CL, Chen KY, Chang CH, Jerng JS, Yu CJ, Yang PC. Timing of tracheostomy as a determinant of weaning success in critically ill patients: a retrospective study. Crit Care. 2005;9(1):R46-52.

29. Stevens RD, Lazaridis C, Chalela JA. The role of mechanical ventilation in acute brain injury. Neurol Clin. 2008;26(2):543-563, x.

30. Bouderka MA, Fakhir B, Bouaggad A, Hmamouchi B, Hamoudi D, Harti A. Early tracheostomy versus prolonged endotracheal intubation in severe head injury. J Trauma. 2004;57(2):251-254.

31. Durbin CG, Jr., Perkins MP, Moores LK. Should tracheostomy be performed as early as 72 hours in patients requiring prolonged mechanical ventilation? Respir Care. 2010;55(1):76-87.

32. Leo P, McCrea M. In: Laskowitz D, Grant G, editors. Translational Research in Traumatic Brain Injury. Boca Raton (FL): CRC Press/Taylor and Francis Group; 2016. Chapter 1.

33. Falcao AL, Dantas Filho VP, Sardinha LA, Quagliato EM, Dragosavac D, Araujo S, Terzi RG. Highlighting intracranial pressure monitoring in patients with severe acute brain trauma. Arq Neuropsiquiatr. 1995;53(3-A):390394.

34. Dantas Filho VP, Falcao AL, Sardinha LA, Facure JJ, Araujo S, Terzi RG. [Relevant factors influencing the evolution of 206 patients with severe head injury]. Arq Neuropsiquiatr. 2004;62(2A):313-318.

35. Oliveira RA, Araujo S, Falcao AL, Soares SM, Kosour C, Dragosavac D, Cintra EA, et al. Glasgow outcome scale at hospital discharge as a prognostic index in patients with severe traumatic brain injury. Arq Neuropsiquiatr. 2012;70(8):604-608.

36. Martins ET, Linhares MN, Sousa DS, Schroeder HK, Meinerz J, Rigo LA, Bertotti MM, et al. Mortality in severe traumatic brain injury: a multivariated analysis of 748 Brazilian patients from Florianopolis City. J Trauma.
2009;67(1):85-90.

37. Kim YJ. A systematic review of factors contributing to outcomes in patients with traumatic brain injury. J Clin Nurs. 2011;20(11-12):1518-1532.

38. Maas AI, Hukkelhoven CW, Marshall LF, Steyerberg EW. Prediction of outcome in traumatic brain injury with computed tomographic characteristics: a comparison between the computed tomographic classification and combinations of computed tomographic predictors. Neurosurgery. 2005;57(6):1173-1182; discussion 1173-1182.

39. Deepika A, Prabhuraj AR, Saikia A, Shukla D. Comparison of predictability of Marshall and Rotterdam CT scan scoring system in determining early mortality after traumatic brain injury. Acta Neurochir (Wien). 2015;157(11):2033-2038.

40. Roquilly A, Cinotti R, Jaber S, Vourc'h M, Pengam F, Mahe PJ, Lakhal K, et al. Implementation of an evidence-based extubation readiness bundle in 499 brain-injured patients. a before-after evaluation of a quality improvement project. Am J Respir Crit Care Med. 2013;188(8):958-966.

41. King CS, Moores LK, Epstein SK. Should patients be able to follow commands prior to extubation? Respir Care. 2010;55(1):56-65.

42. Dunham CM, Ransom KJ. Assessment of early tracheostomy in trauma patients: a systematic review and metaanalysis. Am Surg. 2006;72(3):276-281.

43. Ahmed N, Kuo YH. Early versus late tracheostomy in patients with severe traumatic head injury. Surg Infect (Larchmt). 2007;8(3):343-347.

44. Barquist ES, Amortegui J, Hallal A, Giannotti G, Whinney R, Alzamel H, MacLeod J. Tracheostomy in ventilator dependent trauma patients: a prospective, randomized intention-to-treat study. J Trauma. 2006;60(1):91-97.

45. Huang H, Li Y, Ariani F, Chen X, Lin J. Timing of tracheostomy in critically ill patients: a meta-analysis. PLoS One. 2014;9(3):e92981.

46. Koch T, Hecker B, Hecker A, Brenck F, Preuss M, Schmelzer T, Padberg W, et al. Early tracheostomy decreases ventilation time but has no impact on mortality of intensive care patients: a randomized study. Langenbecks Arch Surg. 2012;397(6):1001-1008. 